\title{
SHM MAPS AND CONJUGATION
}

\author{
MING-JUNG LEE
}

\begin{abstract}
We call two shm maps completely shm homotopic if one map is shm homotopic to a shm conjugation of the other. It is proved that there is a one-to-one correspondence between completely shm homotopy classes of shm maps on $H$-spaces and base point free homotopy classes of maps on their classifying spaces.
\end{abstract}

1. Introduction. In [1], Anderson proved that for discrete groups $G$ and $\bar{G}$, the reduced classifying functor $\bar{B}$ of Segal [3] induces a bijection between the conjugacy classes of homomorphisms $\operatorname{Hom}(G, \bar{G})_{c}$ and the homotopy classes of maps $[\bar{B} G, \bar{B} G]$. On the other hand, Fuchs [2] proved that for associative $\mathrm{CW} H$-spaces (topological monoids with inverses) $H$ and $\bar{H}$, the Dold-Lashof classifying functor $B$ induces a bijection from shm $[H, \bar{H}]_{\pi}$, the set of shm homotopy classes of shm maps, to $[(B H, b),(B \bar{H}, \bar{b})]$, the set of base point preserving homotopy classes of maps. Combining these two cases together, we call two maps between associative $H$-spaces $f$ and $g: H \rightarrow \bar{H}$ completely shm homotopic if there is an inner shm map $\varphi$ on $\bar{H}$ such that $\varphi f$ is shm homotopic to $g$ (for definition of inner shm map see $\S 2$ ). Denote by shm $[H, \bar{H}]_{c}$ the set of completely shm homotopy classes of shm maps. In this paper, we prove

THEOREM 1. Let $H$ and $\bar{H}$ be associative $\mathrm{CW} H$-spaces. The classifying functor $B$ induces a bijection from shm $[H, \bar{H}]_{c}$ to $[B H, B \bar{H}]$.

An important step of the proof is to show that $B$ is a well-defined map between these two sets where we use a generalization of a theorem of Segal [3] to shm maps.

CoROllaRY 2. If $\bar{H}$ is connected, then $\operatorname{shm}[H, \bar{H}]_{\pi} \cong[B H, B \bar{H}]$.

COROLlaRY 3. The category $\mathscr{H}_{c}$ of associative $\mathrm{CW} H$-spaces and completely shm homotopy classes of maps is equivalent to the category $\mathscr{T}$ of connected $\mathrm{CW}$-complexes and (free) homotopy classes of maps.

Presented to the Society, January 25, 1973; received by the editors April 30, 1973.

AMS (MOS) subject classifications (1970). Primary 55D35, 55D45.

Key words and phrases. Shm homotopy, classifying functor, inner shm map, topological category, $H$-spaces.

(C) American Mathematical Society 1974 
Corollary 3 relates results of Anderson and Fuchs, cf. also Stasheff [5, p. 251]. We will use the Dold-Lashof classifying functor $B$ throughout the paper (the classifying functor of Segal [3] will be denoted by $\bar{B}$ for distinction).

2. Inner shm maps and completely shm homotopies. Let $H$ and $\bar{H}$ be associative $H$-spaces. Recall that $f$ is a shm map from $H$ to $\bar{H}$ if $f$ consists of maps $f_{n}: I^{n} \times H^{n+1} \rightarrow \bar{H}$ for $n=0,1, \cdots$ such that

$$
\begin{aligned}
f_{n}\left(h_{0}, t, h_{1}, \cdots, t_{n}, h_{n}\right) & =f_{n-1}\left(\cdots, h_{i-1} h_{i}, \cdots\right) \quad \text { if } t_{i}=0, \\
& =f_{i}\left(h_{0}, t_{1}, \cdots, h_{i-1}\right) f_{n-i}\left(h_{i}, t_{i+1}, \cdots, h_{n}\right) \\
& \text { if } t_{i}=1 .
\end{aligned}
$$

(Note that the $t_{i}$ in this definition is equivalent to $1-t_{i}$ of Fuchs' definition.) We will suppress the subscript of $f$ whenever it is clear from the context. Two shm maps $f$ and $g$ are shm homotopic, denoted by $\simeq_{\text {shm }}$, if there is a continuous family of maps $K_{n}^{t}: I^{n} \times H^{n+1} \rightarrow \bar{H}$ for $t$ in $[0,1]$ such that $K^{t}$ is a shm map and $K^{0}=f, k^{1}=g$. Recall also (cf. Fuchs [2, p. 200]) that, for shm maps $f: H \rightarrow \bar{H}$ and $g: \bar{H} \rightarrow H^{\prime}$, the composition $g f: H \rightarrow H^{\prime}$ is defined by

$$
\begin{aligned}
&(g f)_{0}=f_{0} g_{0}, \\
&(g f)\left(h_{0}, t_{1}, \cdots, t_{n}, h_{n}\right)=g\left(f\left(h_{0}, 2 t_{1}, \cdots, 2 t_{i_{1}}, h_{i_{1}}\right), 2 t_{i_{1}+1}-1,\right. \\
&\left.\cdots, 2 t_{i_{j}+1}-1, f\left(h_{i_{j}+1}, \cdots, 2 t_{n}, h_{n}\right)\right)
\end{aligned}
$$

where $t_{i_{1}+1}, \cdots, t_{i_{j}+1} \in\left[\frac{1}{2}, 1\right]$ and $t_{i} \in\left[0, \frac{1}{2}\right]$ otherwise.

Let $h$ be in $\bar{H}, h^{\prime}$ be a homotopy inverse of $h$ and $l$ be a path from identity $e$ to $h h^{\prime}$. The inner shm map $\varphi_{h^{\prime}, h, l}$ is a shm map from $H$ to $\bar{H}$ with

$$
\left(\varphi_{h^{\prime}, h, l}\right)_{n}\left(h_{0}, t_{1}, \cdots, t_{n}, h_{n}\right)=h^{\prime} h_{0} l\left(t_{1}\right) \cdots l\left(t_{n-1}\right) h_{n} h .
$$

Note $\left(\varphi_{h^{\prime}, h, l}\right)_{0}$ looks like a conjugation. Before we prove that completely shm homotopy, denoted by $\simeq_{c}$, is an equivalence relation, we need a generalization of a theorem of Fuchs [2, p. 203] whose proof is essentially the same as that of Fuchs.

Proposition 4. The shm homotopy class of $\varphi_{h^{\prime}, h, l}$ depends only on the (arc-) component of $h$.

It follows from Proposition 4 that the inner shm map $\varphi_{h^{\prime}, h . l \text {, is shm }}$ homotopic to id ${ }_{H}$ if $h$ is in the identity component of $H$. In general, it is not known when is $\varphi_{h^{\prime}, h, l} \mathrm{shm}$ homotopic to $\mathrm{id}_{H}$. (If $H$ is a discrete abelian group, then of course each $\varphi_{h^{\prime}, h, l}$ is $\mathrm{id}_{H}$.) For any $y$ in the identity component and a path $l$ from $e$ to $y$, one can define a left (right) 
shm translation $L_{y, l}\left(R_{y, l}\right)$ by $\left(L_{y, l}\right)_{n}\left(h_{0}, t_{1}, \cdots, h_{n}\right)=y h_{1} l\left(t_{1}\right) \cdots h_{n}$ $\left(\left(R_{y, l}\right)_{n}\left(h_{0}, t_{1}, \cdots, h_{n}\right)=h_{1} l\left(t_{1}\right) \cdots h_{n} y\right)$. It is obvious to show

LEMMA 5. $L_{y, l}$ and $R_{y, l}$ are shm homotopic to $\mathrm{id}_{H}$.

COROLlaRY 6. The shm homotopy classes of inner shm maps on $H$, under the operation of composition, form a group which is isomorphic to $\pi_{0}(H)$. Consequently, completely shm homotopy is an equivalence relation.

Proof. By Proposition 4 and Lemma 5, one only has to check

where

$$
\varphi_{h_{2}^{\prime}, h_{2}, l_{2}} \circ \varphi_{h_{1}^{\prime}, h_{1}, l_{1}}=\varphi_{h_{2}{ }^{\prime} h^{\prime}, h_{1} h_{2}, l_{3}}
$$

$$
\begin{aligned}
l_{3}(t) & =l_{1}(2 t), & & \text { if } 0 \leqq t \leqq \frac{1}{2}, \\
& =h_{1} l_{2}(2 t-1) h_{1}^{\prime}, & & \text { if } \frac{1}{2} \leqq t \leqq 1 .
\end{aligned}
$$

Q.E.D.

Corollary 7. $\operatorname{shm}(H, \bar{H})_{\pi} / \pi_{0}(\bar{H}) \cong s h m(H, \bar{H})_{c}$.

3. The functors $B$ and $J \Omega$. In [3] Segal proved that, for topological groups, the induced map of an inner automorphism on the classifying space is (free) homotopic to the identity. We will show the same is true for $H$-spaces and inner shm maps. First we observe that shm maps (perhaps now they should be called shm functors) can also be defined between topological categories. Let $C$ and $D$ be topological categories (a topological category is a category whose morphism set is a topological space). We say $f$ is a shm map from $C$ to $D$ if $f$ consists of maps $f_{n}: I^{n} \times C^{n+1} \rightarrow D$ for $n=0,1, \cdots$ where $C^{n+1}=\left\{x_{0}, x_{1}, \cdots, x_{n} \mid x_{0} \cdots x_{n}\right.$ is composable in $\left.C\right\}$ such that

(1) $f_{i}\left(x_{0}, t_{1}, \cdots, t_{i}, x_{i}\right) \cdot f_{j}\left(y_{0}, \cdots, y_{j}\right)$ is composable whenever $x_{i} y_{0}$ is composable.

(2) $f_{n}\left(x_{0}, \cdots, x_{n}\right)=f_{n}\left(\cdots, x_{i-1} x_{i}, \cdots\right)$ if $t_{i}=0$.

(3) $f_{n}\left(x_{0}, \cdots, x_{n}\right)=f\left(x_{0}, \cdots, x_{i-1}\right) f\left(x_{i}, \cdots, x_{n}\right)$ if $t_{i}=1$.

Then same as Sugawara [6] or Fuchs [2] shm maps induce maps between the Dold-Lashof classifying spaces. The following theorem generalizes a theorem of Segal [3].

THEOREM 8. Let $C$ and $D$ be topological categories and let $f, g$ be shm maps from $C$ to $D$. If there is a continuous map $\eta$ from $|C|$, object space of $C$, to $D$ such that

$$
g_{n}\left(h_{0}, \cdots, h_{n}\right) \cdot \eta(c)=\eta\left(c^{\prime}\right) f_{n}\left(h_{0}, \cdots, h_{n}\right) \text { for all }\left(h_{0}, t_{1}, \cdots, h_{n}\right)
$$

where $c=$ domain $h_{n}$ and $c^{\prime}=$ range $h_{0}$. Then $B f \simeq B g$. 
Proof. Consider the category $C \times J$ where $J$ is the ordered set $\{0,1\}$. Define a shm map $F: C \times J \rightarrow D$ by letting

$$
\begin{array}{ll}
F_{n}\left(\left(h_{0}, \alpha_{0}\right), t_{1}, \cdots, t_{n},\left(h_{n}, \alpha_{n}\right)\right) & \\
=\eta\left(c^{\prime}\right) f\left(h_{0}, t_{1}, \cdots, h_{n}\right) & \text { if some } \alpha_{i}=(0,1) \text { the unique morphism } \\
\quad=f\left(h_{0}, t_{1}, \cdots, h_{n}\right) & \text { if all } \alpha_{i}=0 \\
=g\left(h_{0}, t_{1}, \cdots, h_{n}\right) & \text { if all } \alpha_{i}=1 .
\end{array}
$$

It is easy to see that $F_{n}(\cdots)=F_{n-1}\left(\cdots,\left(h_{j-1}, \alpha_{j-1}\right)\left(h_{j}, \alpha_{j}\right), \cdots\right)$ if $t_{j}=0$. When $t_{j}=1$ and some $\alpha_{i}=(0,1)$ we have

$$
\begin{aligned}
F_{j}\left(\left(h_{0}, \alpha_{0}\right), \cdots,\left(h_{j}, \alpha_{j}\right)\right) F_{n-j}\left(\left(h_{j+1}, \alpha_{j+1}\right), \cdots,\left(h_{n}, \alpha_{n}\right)\right) \\
= \\
= \begin{cases}\eta\left(c^{\prime}\right) f\left(h_{0}, \cdots, h_{j}\right) f\left(h_{j+1}, \cdots, h_{n}\right) \quad \text { if } i \leqq j, \\
g\left(h_{0}, \cdots, h_{j}\right) \eta(d) f\left(h_{j+1}, \cdots, h_{n}\right) \quad \text { if } i>j\end{cases} \\
=\eta\left(c^{\prime}\right) f\left(h_{0}, \cdots, h_{j}\right) f\left(h_{j+1}, \cdots, h_{n}\right) \\
=F_{n}\left(\left(h_{0}, \alpha_{0}\right), \cdots,\left(h_{n}, \alpha_{n}\right)\right)
\end{aligned}
$$

where $d=$ domain $h_{j}=$ range $h_{j+1}$. So $F$ is indeed a shm map and it reduces a map from $B(C \times J)$ to $B D$. Although $B C \times I=B C \times B J$ is not homeomorphic to $B(C \times J)$, we only need a map $\mu$ from $B C \times B J$ to $B(C \times J)$ with the properties that

$$
\mu\left(\left(h_{0}, t_{1}, \cdots, h_{n}\right), 0\right)=\left(\left(h_{0}, 0\right), \cdots,\left(h_{n}, 0\right)\right)
$$

and

$$
\mu\left(\left(h_{0}, t_{1}, \cdots, h_{n}\right), 1\right)=\left(\left(h_{0}, 1\right), \cdots,\left(h_{n}, 1\right)\right) .
$$

Recall from Segal [3] that $B(C)=\bar{B}\left(C_{N}\right)$ where $\bar{B}$ is the classifying functor of Segal, $N$ is the set of natural numbers and $C_{N}$ is the subcategory of $N \times C$ obtained by deleting all the morphism of the form $(n, c) \rightarrow\left(n, c^{\prime}\right)$ except the identity morphism. Let $\psi$ be the functor from $H_{N} \times J_{N}$ to $(H \times J)_{N}$ defined by $\psi((i, h),(j, \alpha))=(i+j,(h, \alpha))$ where $i+j$ is the unique morphism from domain $i+$ domain $j$ to Range $i+$ Range $j$. It is easily checked that $\mu=\bar{B} \psi: B H \times B J=\bar{B}\left(H_{N}\right) \times \bar{B}\left(J_{N}\right)=\bar{B}\left(H_{N} \times J_{N}\right) \rightarrow$ $\bar{B}(H \times J)_{N}=B(H \times J)$ has the desired properties. (Note that $\bar{B}$ does commute with products in the category of $k$-spaces.)

COROllary 9. For any inner shm map $\varphi=\varphi_{h^{\prime}, h_{. l}}$ on $H, B \varphi \simeq B_{\mathrm{id}_{B}}$.

ProOf. Let $\varphi^{\prime}$ be $R_{h h^{\prime}, l}$ (see Lemma 5). Then $h^{\prime} \varphi_{n}^{\prime}=\varphi_{n} h^{\prime}$ for all $n$ and the hypothesis of Theorem 8 is satisfied by taking $\eta(H)=h^{\prime}$ where $H$ is now the unique object of the category $H$. Hence, by Theorem 8 , Lemma 5 and a theorem of Fuchs [2, p. 212], we have $B \varphi \simeq B \varphi^{\prime} \simeq{ }_{b} B_{\mathrm{id}_{B}}$ where " $\simeq_{b}$ " means homotopy preserving base point. Q.E.D. 
THEOREM 10. The classifying functor $B$ induces a functor from $\mathscr{H}_{c}$ to $\mathscr{T}$.

Proof. This is a direct consequence of the theorem on p. 212 of Fuchs [2] and Corollary 9.

On the other hand, suppose $\left(X, x_{0}\right)$ and $\left(Y, y_{0}\right)$ are connected base pointed topological spaces. Recall that the Moore loop functor $\Omega$ and the inclusion functor $J: \operatorname{Hom}(H, \bar{H})_{\pi} \rightarrow \operatorname{shm}(H, \bar{H})_{\pi}$ give rise to a map $J \Omega:\left[\left(X, x_{0}\right),\left(Y, y_{0}\right)\right] \rightarrow \operatorname{shm}\left(\Omega\left(X, x_{0}\right), \Omega\left(Y, y_{0}\right)\right)_{\pi}$.

Proposition 11. J $\Omega$ is a well-defined map from $\left[\left(X, x_{0}\right),\left(Y, y_{0}\right)\right]^{\prime}$ to shm $\left(\Omega\left(X, x_{0}\right), \Omega\left(Y, y_{0}\right)\right)_{c}$ where [ , ]' denotes the free homotopy classes of base point preserving maps.

Proof. Let $f$ and $g:\left(X, x_{0}\right) \rightarrow\left(Y, y_{0}\right)$ be maps preserving base point. Let $K: X \times I \rightarrow Y$ be a homotopy from $f$ to $g$. Define a loop $\sigma$ in $\Omega\left(Y, y_{0}\right)$ by $\sigma(t)=K\left(x_{0}, t\right)$. Let $l$ be the path from $\bar{e}$ to $\sigma \cdot \sigma^{-1}$ in $\Omega\left(Y, y_{0}\right)$ such that $l(s)=\sigma_{s} \cdot \sigma_{s}^{-1}$ where $\sigma_{s}=\sigma /[0, s]$. To show $\Omega f$ is completely shm homotopic to $\Omega g$, we observe there is a shm homotopy $\psi$ from $\varphi_{\sigma^{-1, \sigma, l}}^{-1} \circ \Omega f$ to $\Omega g$ as follows.

$$
\psi_{n}^{s}\left(h_{0}, \cdots, h_{n}\right)=\sigma_{s}^{-1} K\left(s, h_{1}\right) l(s t) \cdots l\left(s t_{n-1}\right) K\left(s, h_{n}\right) \sigma_{s}
$$

where $K\left(s, h_{i}\right)(t)=K\left(s, h_{i}(t)\right) \in \Omega\left(Y, y_{0}\right)$. Note that $\varphi \Omega f\left(h_{0}, \cdots, h_{n}\right)=$ $\sigma^{-1} \Omega f\left(h_{1}\right) l\left(t_{1}\right) \cdots l\left(t_{n-1}\right) \Omega f\left(h_{n}\right) \sigma$ since $\Omega f$ is a homomorphism, cf. Fuchs $[2$, p. 200].

4. Proof of Theorem 1. Since $B H$ and $B \bar{H}$ are connected $C W$-complexes, every map from $B H$ to $B \bar{H}$ is homotopic to a map in $[B H, b ; B H, \bar{b}]^{\prime}$, the free homotopy classes of base preserving maps, see Spanier [4, p. 380]. It is sufficient to show $B: \operatorname{shm}(H, \bar{H})_{c} \rightarrow[B H, b ; B \bar{H}, \bar{b}]^{\prime}$ is one-to-one and onto. The ontoness follows from a theorem of Fuchs which says $B: \operatorname{shm}(H, \bar{H})_{\pi} \rightarrow[B H, b ; B \bar{H}, \bar{b}]$ is onto. To show $B$ is one-toone, we suppose $B f \simeq B g$. Then by Proposition 11 we have $\varphi_{\sigma^{-1}, \sigma, l} \circ$ $J \Omega B f \simeq{ }_{\text {shm }} J \Omega B g$. By a theorem of Fuchs [2, p. 215], we have shm isomorphisms $A: H \rightarrow \Omega B H$ and $\bar{A}: H \rightarrow \Omega B \bar{H}$ such that

$g \simeq \simeq_{\mathrm{shm}} \bar{A}^{-1} J \Omega B g A \simeq \simeq_{\mathrm{shm}} \bar{A}^{-1} \varphi J \Omega B f A \simeq \bar{A}^{-1} \varphi \bar{A} \bar{A}^{-1} J \Omega B f A \simeq_{\mathrm{shm}} \bar{A}^{-1} \varphi \bar{A} f$.

In order to show $f \simeq_{c} g$, it now only remains to prove $\bar{A}^{-1} \varphi \bar{A}$ is shm homotopic to an inner shm homomorphism on $\bar{H}$. First observe that we may assume $\bar{A}^{-1}$ carries identity to identity, see Fuchs [2, pp. 215, 222]. Let $l^{\prime}$ be the path in $\bar{H}$ such that $l^{\prime}(t)=\bar{A}^{-1}(l(2 t))$ if $0 \leqq t \leqq \frac{1}{2}$ and $l^{\prime}(t)=$ $\bar{A}^{-1}\left(\sigma, 2 t-1, \sigma^{-1}\right)$ if $\frac{1}{2} \leqq t \leqq 1$. Then $l^{\prime}$ is a path from $\bar{e}$ to $h h^{\prime}$ where 
$h=\bar{A}^{-1}(\sigma)$ and $h^{\prime}=\bar{A}^{-1}\left(\sigma^{-1}\right)$. Denote $\varphi_{h^{\prime} h, l^{\prime}}$ by $\varphi^{\prime}$. The proof will be completed if we show the following diagram is shm homotopy commutative.

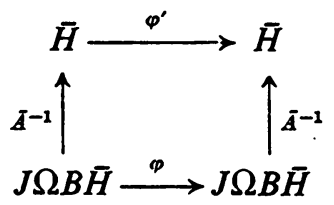

Recall the definition of composition of shm maps, we have

$$
\begin{aligned}
& \left(\bar{A}^{-1} \varphi\right)_{n}\left(h_{0}, t_{1}, \cdots, t_{n}, h_{n}\right) \\
& =\bar{A}^{-1}\left(\sigma^{-1} h_{0} l\left(2 t_{1}\right) \cdots l\left(2 t_{i}\right) h_{i} \sigma, 2 t_{i_{1}+1}-1, \cdots,\right. \\
& \left.2 t_{i,+1}-1, \sigma^{-1} h_{i,+1} \cdots l\left(2 t_{n}\right) h_{n} \sigma\right), \\
& \left(\varphi^{\prime} \bar{A}^{-1}\right)_{n}\left(h_{0}, t_{1}, \cdots, t_{n}, h_{n}\right) \\
& =h^{\prime} \bar{A}^{-1}\left(h_{0}, 2 t_{1}, \cdots, 2 t_{i_{1}}, h_{i_{1}}\right) l^{\prime}\left(2 t_{i_{1}+1}-1\right) \cdots \\
& l^{\prime}\left(2 t_{i_{j}+1}-1\right) \bar{A}^{-1}\left(h_{i,+1}, \cdots, 2 t_{n}, h_{n}\right) h
\end{aligned}
$$

where $t_{i_{1}+1}, \cdots, t_{i_{j}+1} \in\left[\frac{1}{2}, 1\right]$ and $t_{i} \in\left[0, \frac{1}{2}\right]$ otherwise (note again our $t_{i}$ is equivalent to Fuchs' $1-t_{i}$ ). Finally we leave to the reader to check the following map $\psi$ is a shm homotopy from $\bar{A}^{-1} \varphi$ to $\varphi^{\prime} \bar{A}^{-1}$, i.e. $\psi^{0}=\bar{A}^{-1} \varphi$ and $\psi^{1}=\varphi^{\prime} \bar{A}^{-1}$.

$$
\begin{array}{r}
\psi_{n}^{s}\left(h_{0}, t_{1}, \cdots, t_{n}, h_{n}\right) \\
=\bar{A}^{-1}\left(\sigma^{-1}, s, h_{0}, s 2 t_{1}, l\left((1-s) 2 t_{1}\right) h_{1}, \cdots, 2 t_{i 1}, l\left((1-s) 2 t_{i_{1}}\right) h_{i_{1}}, s\right. \\
Q^{s}\left(2 t_{i_{1}+1}-1\right), s, \cdots, s, Q^{s}\left(2 t_{i j+1}-1\right), s, h_{i j+1}, \\
\left.s 2 t_{i j+2}, \cdots, s 2 t_{n}, l\left((1-s) 2 t_{n}\right) h_{n}\right)
\end{array}
$$

where $Q^{s}: I \rightarrow \bar{H} \times I \times \bar{H}$ and

$$
\begin{aligned}
Q^{s}(t) & =(\bar{e}, 0, l(1-s+2 t)) & & \text { if } 2 t \leqq s \\
& =\left(\sigma,(2 t-s) /(2-s), \sigma^{-1}\right) & & \text { if } s \leqq 2 t
\end{aligned}
$$

In general $Q^{s}$ may not be a well-defined map, but it is well defined when composing with $\bar{A}_{n}^{-1}$ because $\bar{A}_{n}^{-1}\left(\cdots, Q^{s}(t), \cdots\right)=\bar{A}_{n-1}^{-1}\left(\cdots, \sigma \sigma^{-1}, \cdots\right)$ when $s=2 t$. Note also that $\bar{A}_{1}^{-1} Q^{1}(t)=l^{\prime}(t)$ and $Q^{0}(t)=\left(\sigma, t, \sigma^{1}\right)$. Q.E.D.

In the previous proof we have in fact proved

Proposition 12. If $A: H \rightarrow \bar{H}$ is a shm isomorphism, then it carries homotopy classes of inner shm maps on $H$ to homotopy classes of inner shm maps on $\bar{H}$.

Corollary 2 is a consequence of Theorem 1 and Proposition 4. 


\section{REFERENCES}

1. D. W. Anderson, Simplicial K-theory and generalized homology theories. I (preprint).

2. M. Fuchs, Verallgemeinerte Homotopie-Homomorphismem und Klassifizierde Räume, Math. Ann. 161 (1965), 197-230. MR 33 \#3295.

3. G. Segal, Classifying spaces and spectral sequences, Inst. Hautes Études Sci. Publ. Math. No. 34 (1968), 105-112. MR 38 \#718.

4. E. H. Spanier, Algebraic topology, McGraw-Hill, New York, 1966. MR 35 \#1007. 5. J. Stasheff, H-spaces and classifying spaces. I, Proc. Sympos. Pure Math., vol. 22, Amer. Math. Soc., Providence, R.I., 1971, p. 247.

6. M. Sugawara, On the homotopy-commutativity of groups and loop spaces, Mem. Coll. Sci. Univ. Kyoto Ser. A Math. 33 (1960, 1961), 257-269. MR 22 \#11394.

Department of Mathematics, loutsiana State University, New Orleans, LOUISTANA 70122

Current address: Room 940, South Central Bell, 3300 West Esplanade Avenue, Metairie, Louisiana 70035 